\title{
Three dimensional simulations of Hall magnetohydrodynamics
}

\author{
Daniel O. Gómez ${ }^{1,2}$ \\ ${ }^{1}$ Instituto de Astronomía y Física del Espacio, C.C. 67 - Suc. 28, \\ (1428) Buenos Aires, Argentina \\ email: gomez@iafe.uba.ar \\ ${ }^{2}$ Departamento de Física, Facultad de Ciencias Exactas y Naturales (UBA), \\ Ciudad Universitaria, (1428) Buenos Aires, Argentina
}

\begin{abstract}
Turbulent flows take place in a large variety of astrophysical objects, and often times are the source of dynamo generated magnetic fields. Much of the progress in our understanding of dynamo mechanisms, has been made within the theoretical framework of magnetohydrodynamics (MHD). However, for sufficiently diffuse media, the Hall effect eventually becomes non-negligible.

We present results from simulations of the Hall-MHD equations. The simulations are performed with a pseudospectral code to achieve exponentially fast convergence. We study the role of the Hall effect in the dynamo efficiency for different values of the Hall parameter.
\end{abstract}

Keywords. ISM: magnetic fields, MHD, turbulence.

\section{Introduction}

Numerical simulations have progressively become an important tool to study astrophysical flows. The large-scale dynamics of plasma flows can often be described within a fluidistic approximation known as one-fluid magnetohydrodynamics (MHD). Complex flows such as those corresponding to turbulent regimes are ubiquitous in astrophysics, which is consistent with their extremely large Reynolds numbers.

However, Hall currents can play a significant role in the dynamics of low density and/or low temperature astrophysical plasmas, such as dense molecular clouds (Wardle \& Ng, 1999), accretion disks (Balbus \& Terquem, 2001, Sano \& Stone, 2002), white dwarfs and neutron stars (Yakovlev \& Urpin, 1980) and in reconnection events at the Earth's magnetotail (Mozer, Bale \& Phan, 2002).

In these plasmas the one-fluid MHD description needs to be extended to the so-called two-fluid MHD or Hall MHD. One of the interesting features of the Hall-MHD description, is that in the ideal limit (i.e. without resistivity) the magnetic field is stretched by the electron velocity field rather than the bulk velocity field. Since these two velocity fields can be quite different, the Hall term is expected to produce a measurable effect. For instance, the Hall term increases the reconnection rate with respect to purely MHD regimes (Ma \& Bhattacharjee, 2001, Morales et al., 2005). Also, the Hall term was found to enhance or suppress dynamo action depending on the relative importance of the Hall term when compared to the inductive term (Mininni, Gómez, \& Mahajan, 2002). We therefore perform 3D numerical simulations of the Hall-MHD equations to explore the role of the Hall current in astrophysical scenarios.

In Section 2 we describe the so-called Hall-MHD equations, which stem from a two-fluid description of the plasma. In Section 3 we briefly describe simulations in 2.5 dimensions to study Hall magnetic reconnection. In Section 4 we present the results from $3 \mathrm{D}$ simulations of the Hall-MHD equations to study the efficiency of a turbulent dynamo. Finally, in Section 5 we list our conclusions. 

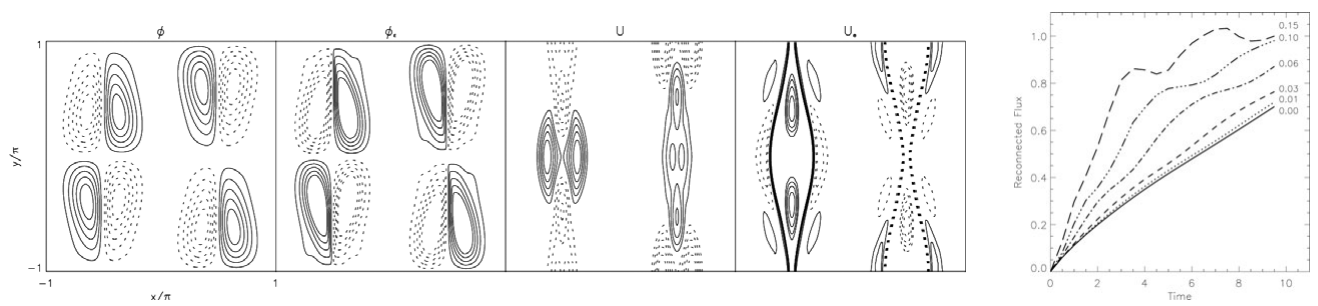

Figure 1. (a) From left to right, contour plots of the proton $(\phi)$ and electron $\left(\phi_{e}\right)$ stream functions, and the out of plane proton $(u)$ and electron $\left(u_{e}\right)$ velocity components. (b) Reconnected magnetic flux vs. time for different values of the Hall parameter $\epsilon$ (labelled).

\section{The Hall-MHD equations}

Highly conductive plasmas tend to develop thin and intense current sheets in their reconnection layers. Whenever the width of the curent sheets reaches values as low as $c / w_{p i}$ ( $w_{p i}$ is the ion plasma frequency and $c$ is the speed of light), it is no longer possible to neglect the Hall term in Ohm's law (Ma \& Bhattacharjee, 2001). For a fully ionized plasma of protons and electrons, the generalized Ohm's law can be written as:

$$
\boldsymbol{E}+\frac{1}{c} \boldsymbol{v} \times \boldsymbol{B}=\frac{1}{\sigma} \boldsymbol{j}+\frac{1}{n e}\left(\frac{1}{c} \boldsymbol{j} \times \boldsymbol{B}-\nabla p_{e}\right),
$$

where $n$ is the electron and proton density, $e$ is the electron charge, $\sigma$ is the electric conductivity, $\boldsymbol{v}$ is the plasma flow velocity, and $\boldsymbol{j}$ is the electric current density. Assuming incompressibility, the Hall-MHD equations can be cast in their dimensionless form as:

$$
\begin{gathered}
\partial_{t} \boldsymbol{v}+(\boldsymbol{v} \cdot \nabla) \boldsymbol{v}=(\nabla \times \boldsymbol{B}) \times \boldsymbol{B}-\nabla p+\nu \nabla^{2} \boldsymbol{v}, \\
\partial_{t} \boldsymbol{B}=\nabla \times[(\boldsymbol{v}-\epsilon \nabla \times \boldsymbol{B}) \times \boldsymbol{B}]+\eta \nabla^{2} \boldsymbol{B}, \\
\nabla \cdot \boldsymbol{B}=0=\nabla \cdot \boldsymbol{v} .
\end{gathered}
$$

In Eqs (2.2)-(2.4) we have normalized $\boldsymbol{B}$ and $\boldsymbol{v}$ to the Alfvén speed $v_{A}=B_{0} / \sqrt{4 \pi \rho}\left(B_{0}\right.$ : magnetic field intensity, $\rho$ : mass density), the total gas pressure $p$ to $\rho v_{A}^{2}$, and longitudes and times respectively to $L_{0}$ and $L_{0} / v_{A}$. The dimensionless dissipation coefficients are the viscosity $\nu$ and the electric resistivity $\eta$ defined as $\eta=c^{2} /\left(4 \pi \sigma L_{0} v_{A}\right)$. The dimensionless coefficient $\epsilon=c /\left(w_{p i} L_{0}\right)$ is a measure of the relative strength of the Hall effect. The dimensionless electron velocity is:

$$
\boldsymbol{v}_{\boldsymbol{e}}=\boldsymbol{v}-\epsilon \nabla \times \boldsymbol{B} .
$$

From Eqn (2.3) it is apparent that in the non-dissipative limit (i.e. $\eta \rightarrow 0$ ) the magnetic field remains frozen to the electron flow $\boldsymbol{v}_{\boldsymbol{e}}$ rather than to the bulk velocity $\boldsymbol{v}$.

\section{Hall reconnection}

To study the role of the Hall effect on magnetic reconnection, we performed simulations in 2.5 dimensions, i.e. assuming translational symmetry along the cartesian $\hat{\boldsymbol{z}}$-direction. Under this geometrical assumption, the solenoidal fields $\boldsymbol{B}$ and $\boldsymbol{v}$ can be cast:

$$
\begin{aligned}
& \boldsymbol{B}=\nabla \times[\hat{\boldsymbol{z}} a(x, y, t)]+\hat{\boldsymbol{z}} b(x, y, t), \\
& \boldsymbol{v}=\nabla \times[\hat{\boldsymbol{z}} \phi(x, y, t)]+\hat{\boldsymbol{z}} u(x, y, t),
\end{aligned}
$$

where $a$ is the magnetic flux function, $\phi$ is the stream function, and $b$ and $u$ are the $\hat{z}$-components of these fields. The computation is carried out in a rectangular domain assuming periodic boundary conditions and the nonlinear terms are evaluated following 

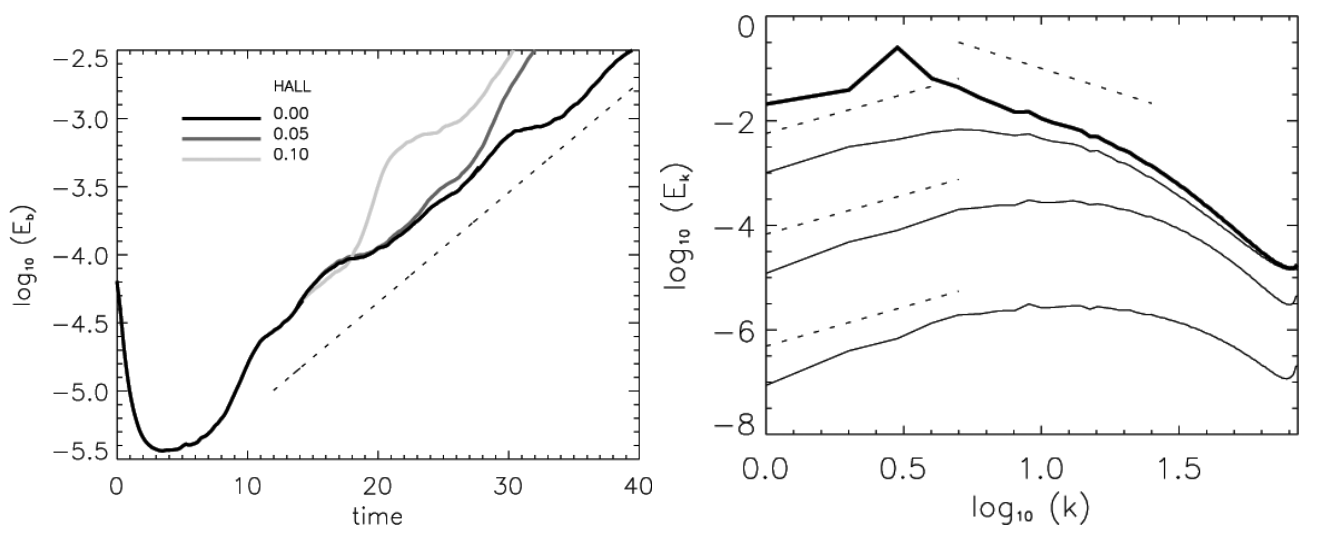

Figure 2. (a) Magnetic energy vs. time for different values of the Hall parameter (labelled). (b)Total energy spectrum (thick trace) at $t=72$ for the case $\epsilon=0.10$. Magnetic energy spectra at $t=18,36,72$ (from bottom to top) are also shown. The Kolmogorov and Kazantsev spectra are overlaid (dotted trace) for reference.

a 2/3 dealiased pseudospectral technique. To provide a reconnection scenario, the initial condition corresponds to two oppositely oriented current sheets to satisfy periodicity (see also Morales et al., 2006). We performed numerical simulations with a spatial resolution of $512 \times 512$ grid points, and different values of the Hall parameter $\epsilon$. The dissipation coefficients are set to $\eta=\nu=0.01$ to ensure that all the lengthscales are properly resolved. Note that pseudospectral methods conserve the energy of the system, i.e. no numerical dissipation is artificially added to the simulation.

The first two panels of Figure 1a show contour plots of the proton $(\phi)$ and electron $\left(\phi_{e}=\phi-\epsilon b\right)$ stream functions at $t=1$. The difference between these patterns contributes to the in-plane electric current density, which in turn generates the out-of-plane magnetic field $b$. The two right hand panels in Figure 1a show contours of the out-of-plane velocities of protons $(u)$ and electrons $\left(u_{e}=u-\epsilon j\right)$. The two species show entirely different velocity patterns. Note that in the ideal limit the magnetic field remains frozen to the electron flow, which is faster than the proton flow. The magnetic flux reconnected as a function of time at the $\mathrm{X}$ point can be calculated in terms of the difference of the magnetic potential in the X-point and the O-point, i.e. $a_{X}(t)-a_{O}(t)$. The effect of the Hall term on the reconnected flux is shown in Figure 1b. As $\epsilon$ is increased the reconnection process becomes more efficient, as evidenced by the total reconnected flux.

\section{Hall dynamo}

Haugen, Brandenburg, \& Dobler (2004) have shown that not only helical flows can generate magnetic fields, but also non-helical flows are able to produce the so-called smallscale dynamos. To study the role of the Hall effect on the generation of magnetic fields by non-helical turbulent flows, we performed 3D simulations of the Hall MHD equations (i.e. Eqs (2.2)-(2.3)) with $256^{3}$ spatial resolution. We first generate a stationary and non-helical hydrodynamic turbulence by applying an external forcing. In a second stage, a random and small magnetic field is introduced at small scales. The exponentially fast growth of magnetic energy is displayed in Figure 2a for runs with different values of the Hall parameter (labelled) (see also Gómez, Mininni, \& Dmitruk, 2010). There is an initial linear stage for which the runs with $\epsilon=0.05$ (moderate Hall) and $\epsilon=0.10$ (large Hall) are indistinguishable from the case with $\epsilon=0.00$ (purely MHD). The slope for these three runs during this stage is indicated by the dotted straight line in Fig. 2a, which corresponds to the growth rate of the MHD case (i.e. $\epsilon=0.00)$. Later in time the run with large Hall 
$(\epsilon=0.10)$ departs from this linear regime and starts growing faster. A similar behavior is observed somewhat later for the run corresponding to moderate Hall $(\epsilon=0.05)$. Therefore, in non-helical flows the Hall effect enhances the dynamo efficiency causing magnetic energy to grow super-exponentially, as has also been observed for large-scale dynamos in helical flows (Mininni, Gómez, \& Mahajan, 2005, also Gómez, \& Mininni, 2004, Mininni, Gómez, \& Mahajan, 2003). The growth of the magnetic energy spectrum is shown in Figure 2b. The Kazantsev slope $E_{k} \propto k^{3 / 2}$ (Kazantsev, 1968) provides a reasonable approximation at small wavenumbers for all these cases, while the kinetic energy spectrum remains always close to Kolmogorov (i.e. $E_{k} \propto k^{-5 / 3}$ ). At saturation, the total magnetic energy reaches a sizeable fraction of the total kinetic energy (15\% to $20 \%$ ). Furthermore, magnetic energy remains smaller than kinetic energy at all spatial scales, which is to be expected for small-scale dynamos.

\section{Conclusions}

In the present paper, we call the attention on the potential relevance of the Hall effect in the dynamics of a number of astrophysical flows, specially those characterized by low electron densities. We quantitatively assess the role of the Hall effect in a number of astrophysical applications, by performing numerical integrations of the Hall MHD equations. As a first application we study magnetic reconnection in $2.5 \mathrm{D}$ simulations, showing the enhancement of the reconnection rate caused by the Hall effect. Gómez, Dmitruk, \& Mahajan (2008) have also extended the so-called reduced MHD equations to include the Hall term (see also Martín, Dmitruk, \& Gómez, 2010), which is the relevant approximation in plasma configurations with a strong external magnetic field. We also studied the role of the Hall effect in the generation of magnetic fields by turbulent nonhelical flows. We show that even though the Hall term does not affect the kinematic dynamo stage during which the magnetic energy grows exponentially fast, it is responsible for a subsequent non-linear state (in between the kinematic and saturation stages) during which the magnetic energy grows super-exponentially fast.

\section{References}

Balbus, S. A. \& Terquem, C. 2001, ApJ, 552, 235.

Gómez, D. O., Mininni, P. D., \& Dmitruk, P. 2010, Phys. Rev. E, 82, 036406.

Gómez, D. O., Dmitruk, P., \& Mahajan, S. M. 2008, Phys. Plasmas, 15, 102303.

Gómez, D. O. \& Mininni, P. D. 2004, Nonlin. Proc. Geophys., 11, 619.

Haugen, N. E. L., Brandenburg, A., \& Dobler, W. 2004, Phys. Rev. E 70, 016308.

Kazantsev, A. P. 1968, Sov. Phys. JETP 26, 1031.

Ma, Z. \& Bhattacharjee, A. 2001, J. Geophys. Res., 106, 3773.

Martín, L., Dmitruk, P., \& Gómez, D. O. 2010, Phys. Plasmas, 17, 112304.

Mininni, P. D., Gómez, D. O., \& Mahajan, S. M. 2005, ApJ, 619, 1019.

Mininni, P. D., Gómez, D. O., \& Mahajan, S. M. 2003, ApJ, 587, 472.

Mininni, P. D., Gómez, D. O., \& Mahajan, S. M. 2002, ApJ, 567, L81.

Morales, L., Gómez, D. O., Dasso, S., \& Mininni, P. D. 2006, Adv. Space Res., 37, 1287.

Morales, L., Dasso, S., Gómez, D. O., \& Mininni, P. D. 2005, J. Atm. Sci. E Sol. Terr. Phys., 67, 1865.

Mozer, F., Bale, S., \& Phan, T. D. 2002, Phys. Rev. Lett., 89, 015002.

Sano, T. \& Stone, J. M. 2002, ApJ, 570, 314.

Wardle, M. \& Ng, C. 1999, Mon. Not. R. A. S., 303, 239.

Yakovlev, D. G. \& Urpin, V. A. 1980, Astr. Zh., 57, 526. 